
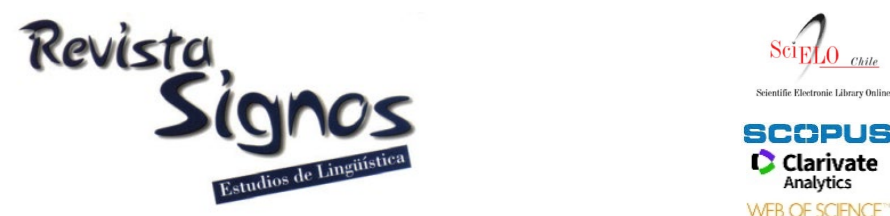

\title{
Estrategias discursivas para la evaluación negativa en comentarios escritos de docentes universitarios*
}

\author{
Discourse strategies for negative evaluations in university \\ professors' written comments
}

\author{
Lucía Natale \\ UNIVERSIDAD NACIONAL DE GENERAL SARMIENTO \\ ARGENTINA \\ lnatale@campus.ungs.edu.ar
}

Recibido: 27-IX-2019 / Aceptado: 19-X-2020

DOI: $10.4067 /$ S0718-09342021000100236

\section{Resumen}

Los comentarios escritos que los docentes universitarios realizan sobre los textos de sus estudiantes siguen, fundamentalmente, dos propósitos: expresar una evaluación (positiva o negativa) y retroalimentar la producción escrita (Kumar \& Strake, 2011). En este último caso, los profesores ofrecen orientaciones e indican qué ajustes pueden introducirse para que el escrito se acerque a las formas aceptadas en una cultura disciplinar. Las evaluaciones, a su vez, pueden estar más o menos abiertas a la negociación, según los recursos discursivos seleccionados para su construcción. Partiendo de los aportes de la Lingüística Sistémico Funcional (Halliday, 1994; Martin \& White, 2005), este artículo presenta un análisis cualitativo de tres estrategias discursivas (ED) (Menéndez, 2005) empleadas por docentes universitarios en comentarios escritos que expresan evaluaciones negativas sobre las producciones de sus estudiantes. Las estrategias -que hemos denominado 'Rechazar', 'Ajustar' y 'Problematizar'- construyen, respectivamente, evaluaciones que responden a las escalas (Thompson \& Hunston, 2000) de lo correcto/incorrecto, preciso/impreciso e incuestionable/cuestionable, que se refieren concretamente a los contenidos conceptuales y a la construcción de saberes por parte de los estudiantes. Para su reconocimiento, hemos considerado los saberes evaluados (términos, datos fácticos, metodología de investigación, por ejemplo) y la manera en que se negocian los posicionamientos intersubjetivos (Martin \& White, 2005). Los resultados encontrados muestran que las evaluaciones negativas ofrecen mayor o menor margen a la negociación, de acuerdo con el tipo de conocimiento evaluado.

Palabras Clave: Escritura académica, comentarios escritos, evaluación negativa, estrategias discursivas, negociación. 


\begin{abstract}
The written comments university professors make about students' texts serve, fundamentally, two purposes: to express an evaluation (positive or negative) and feedback on written production (Kumar \& Strake, 2011). In the latter case, professors offer guidance and indicate what adjustments can be made for the text to meet the disciplinary culture requirements. Evaluations, in turn, can be more or less open to negotiation depending on the discursive resources selected for their construction. Based on the contributions of Systemic Functional Linguistics (Halliday, 1994; Martin \& White, 2005), this paper presents a qualitative analysis of three discourse strategies (Menéndez, 2005) used by professors to express a negative evaluation about their students' productions. The strategies -which we have called 'Rejection', 'Adjustment' and 'Problematization'- construct, respectively, evaluations that respond to scales (Thompson \& Hunston, 2000) such as correct/incorrect, accurate/inaccurate or questionable/unquestionable, which refer, specifically, to the conceptual contents and knowledge construction made by students. In order to identify the strategies mentioned, we have considered the type of knowledge (e. g., terms, factual data, research methods) assessed by professors and the way in which intersubjective positions are negotiated (Martin \& White, 2005). The results show that negative evaluations offer more or less margin for negotiation, according to the type of knowledge evaluated.
\end{abstract}

Key Words: Academic writing, written comments, negative evaluation, discourse strategies, negotiation.

\title{
INTRODUCCIÓN
}

Los comentarios escritos (CE) que los docentes universitarios anotan en los márgenes de las producciones académicas de sus estudiantes conforman uno de los pasos (Martin \& Rose, 2008) que componen el género Devolución Escrita (DE) (Lea \& Street, 1998; Hyland \& Hyland, 2006; Natale, 2013). Los CE, que han recibido reciente atención en el ámbito latinoamericano (Natale, 2013, 2014; Padilla \& López, 2019; Arancibia Gutiérrez, Tapia Ladino \& Correa Pérez, 2019) tienen como función evaluar segmentos específicos de la producción de los alumnos y fundamentar la calificación del escrito (Natale, 2013).

En este sentido, las DE conforman un género evaluativo que, al igual que otros del discurso académico, como el informe de arbitraje entre pares (IAP) (Bolívar, 2008, 2011), es un género de circulación restringida en una esfera. A diferencia de los IAP dirigidos a pares- las DE son un tipo de evaluación intergeneracional, ya que son producidas por un miembro experto y están dirigidas a un novicio que aspira a ingresar a una cultura disciplinar. Así, se caracterizan por las diferencias de status (Poynton, 1985) entre los roles de los participantes en la institución. En este entramado de relaciones intersubjetivas (Martin \& White, 2005), los docentes pueden construir para sí dos papeles: el de gatekeeper (Berkenkotter \& Huckin, 1995) o guardián de la cultura disciplinar -si se erigen como jueces que se limitan a habilitar o impedir el ingreso de los novatos- o el de guía o mentor -si ofrecen retroalimentación 
(Kumar \& Strake, 2011) para que los estudiantes mejoren sus producciones (Natale, 2013).

Las evaluaciones expresadas en los CE pueden ser positivas o negativas. Para ello, los docentes escogen recursos semánticos para dar cuenta de sus evaluaciones y:

"adoptar posiciones, construir personas textuales o identidades discursivas, asumir roles, negociar relaciones y transformar en 'naturales' las posturas intersubjetivas que son, en última instancia, ideológicas" (Kaplan, 2004: 53).

A menudo, la negociación de estas relaciones exige emplear estrategias discursivas (ED) (Menéndez, 2005) que permiten alcanzar simultáneamente finalidades interaccionales que, a primera vista, podrían parecer contrapuestas, como la expresión de una evaluación negativa y el establecimiento de un vínculo de solidaridad entre docentes y estudiantes. Se evita de esta manera poner en riesgo las relaciones entre los participantes del intercambio en un contexto institucional formal.

Este artículo tiene como objetivo presentar un análisis cualitativo, de tipo exploratorio, de tres estrategias discursivas empleadas por docentes universitarios para expresar evaluaciones negativas en CE referidos a producciones de estudiantes prontos a graduarse. Estas ED, que hemos denominado 'Rechazar', 'Ajustar' y 'Problematizar', construyen, respectivamente, evaluaciones que responden a las escalas (Thompson \& Hunston, 2000) de lo correcto/incorrecto, preciso/impreciso e incuestionable/cuestionable, que se refieren a los contenidos conceptuales y a la construcción de saberes por parte de los novatos. Para el análisis, importa discernir el tipo de conocimientos (uso de la terminología, datos fácticos, metodología de la investigación, por ejemplo) evaluados y la manera en que se negocian los posicionamientos intersubjetivos. Asimismo, en estas estrategias, consideramos de qué manera aparecen representados el docente, los estudiantes y la escritura. Entendemos que este estudio resulta de particular interés, ya que permite observar de qué manera se entretejen los vínculos entre miembros de distintas generaciones de una cultura disciplinar y brinda la posibilidad de reconocer recursos del orden discursivosemántico (Martin \& Rose, 2003) que los profesores emplean para expresar su evaluación en un ámbito que requiere del mantenimiento de un orden institucional. A la vez, aporta a la pedagogía de la escritura en el nivel superior. En lo que sigue, tras una breve presentación de los conceptos que guían el análisis, se exponen los pasos metodológicos seguidos y, en la sección Resultados, se examinan los recursos que intervienen en la construcción de las ED 'Rechazar', 'Ajustar' y 'Problematizar'. Finalmente, se presentan algunas conclusiones. 


\section{Marco teórico}

El estudio toma como marco teórico general la Lingüística Sistémico Funcional (LSF), que ofrece ricas reflexiones sobre el rol del lenguaje en la cultura, la vida social y el desarrollo del sujeto. Brinda además herramientas para identificar los recursos lingüísticos que cimentan la construcción de la experiencia, las relaciones interpersonales y la organización de todo evento comunicativo. En este encuadre, son imprescindibles las obras de Michael Halliday como la Introducción a la Gramática Funcional (Halliday, 1994), que provee de categorías analíticas específicas para identificar la entidad o la cláusula objeto de comentario del docente universitario. Allí también se presenta un esquema que permite examinar los intercambios entre profesores y estudiantes a partir de los roles y funciones de habla, aunque en diversas exploraciones iniciales del corpus de devoluciones escritas encontramos que ese esquema no resultaba lo suficientemente fructífero porque se refiere al nivel clausal. A partir del reconocimiento de que los CE constituyen unidades discursivas, incorporamos otros aportes de la LSF, más específicamente, la Teoría de la Valoración, que brinda herramientas específicas para el examen de la metafunción interpersonal en el plano semántico-discursivo (Martin \& White, 2005). También se incorporaron contribuciones de la sociolingüística interaccional (Gumperz, 1982; Brown \& Levinson, 1987; Tannen, 1993), de la pragmática (Sperber \& Wilson, 1986) y del análisis pragmático del discurso (Menéndez, 2005), en las que se reconocen las intenciones de los participantes de la interacción. Estas líneas teóricas coinciden en un aspecto que resulta fundamental a los fines de este estudio, ya que parten de la consideración del contexto en que se producen los intercambios.

Una noción central para nuestro análisis es la de Estrategia discursiva (ED). Una estrategia discursiva implica una reconstrucción analítica de un plan de acción que el hablante/escritor en tanto sujeto discursivo pone en funcionamiento combinando recursos gramaticales y discursivos para alcanzar una finalidad interaccional (Menéndez, 2005). Hablamos de reconstrucción analítica en tanto no podemos asegurar que lo que se realiza es lo que el hablante ha planificado expresar, sino lo que surge del análisis de su discurso. En esta línea, la tarea del analista consiste en la reconstrucción de las distintas ED que los hablantes en tanto sujetos discursivos emplean para lograr o intentar lograr sus fines comunicativos (Menéndez, 2005), tratando de preservar su imagen y la de sus destinatarios (Brown \& Levinson, 1987).

\subsection{La Teoría de la Valoración}

En el marco de la LSF, las relaciones entre los participantes de una situación se corresponden con la variable Tenor del Registro (Halliday, 1978), una dimensión semiótica en la que se negocian las relaciones sociales (Martin, 1992), que se realizan en el lenguaje a través de la metafunción interpersonal, vinculada con la expresión del 
hablante, sus actitudes y juicios, así como sus relaciones sociales. El Tenor de las relaciones puede considerarse desde tres variables: el Status (o Poder), el Contacto y el Afecto (Poynton, 1985). Para este trabajo nos importa especialmente el Status, relativo a las posiciones jerárquicas que los participantes de la interacción ostentan en el marco de una cultura y, más concretamente, en los círculos sociales en los que actúan. Se trata de un posicionamiento relativo, otorgado en general por las instituciones. Los participantes de una situación pueden establecer entonces relaciones de de simetría o asimetría (Martin \& White, 2005).

La expresión de la subjetividad del hablante/escritor, su sistema de valores, su opinión y sentimientos acerca de aquello a lo que se está refiriendo constituye un aspecto sumamente importante en el estudio del lenguaje, por lo que ha sido abordado por distintos autores (Halliday, 1994; Thompson \& Hunston, 2000; Martin \& White, 2005, entre muchos otros).

En cuanto a lo que constituye objeto de opinión o evaluación, este puede ser o bien una entidad (designada por un sintagma nominal) o bien una proposición (cláusula). En la tradición de la LSF, cada uno de estos objetos de evaluación son considerados por medio de dos conceptos distintos: los significados actitudinales, que refieren a la evaluación de las entidades, y la modalidad, relativa a la evaluación de las proposiciones y propuestas (Halliday, 1994). A su vez, los recursos evaluativos se organizan en escalas como la de la bondad/maldad, certeza/falsedad, relevancia/irrelevancia, por ejemplo, aunque todas se pueden subsumir en la primera, la más general (Hunston \& Thompson, 2000).

A través de las valoraciones empleadas por un hablante, este se posiciona intersubjetivamente en el discurso y negocia significados interpersonales (Hood \& Martin, 2005; Martin \& White, 2005). Así, los recursos seleccionados sirven para reconocer su sistema de valores y los de su comunidad, además de construir lazos entre interlocutores y organizar el discurso (Hunston \& Thompson, 2000).

La puesta en juego de las relaciones interpersonales en la evaluación puede constituir la causa de las dificultades que acarrea su análisis, dado que no existe un conjunto discreto de recursos, sean estos gramaticales o léxicos, que estén enteramente al servicio de las funciones mencionadas, más allá de que los adjetivos y los adverbios suelen ser sus vías de expresión. De hecho, es frecuente que la evaluación no se manifieste explícitamente, sino a través de una serie de elementos que la sugieren. Por ello, resulta necesario apelar a nociones discursivas que contemplen el interjuego producido en el contexto en que los enunciados son producidos e interpretados, así como el entramado de relaciones intersubjetivas construido con los recursos evaluativos. En este sentido, adquieren relevancia los aportes de la Teoría de la Valoración (Hood \& Martin, 2005; Martin \& White, 2005) y 
del reconocimiento del dialogismo presente en toda producción lingüística, de acuerdo con Bajtín.

La Teoría de la Valoración plantea una división de los recursos evaluativos en tres grandes dominios semánticos o subtipos de valoración: Actitud, Compromiso y Gradación. La Actitud refiere a los significados a través de los cuales los escritores/hablantes evalúan intersubjetivamente a los participantes y los procesos en términos de emociones (Afecto), normas sociales (Juicios) y principios estéticos o sistemas de valor social (Apreciación).

El Compromiso permite interpretar el origen de los sentimientos que se negocian. Desde una perspectiva bajtiniana, se reconoce una relación con otras voces y se establece una sub-clasificación de enunciados: monoglósicos y heteroglósicos. Los primeros ignoran la diversidad de posiciones, por lo que se utilizan recursos como enunciados declarativos, mientras que en los segundos se reconoce la diversidad de posiciones y se emplean recursos de los sistemas de proyección, modalidad y concesión (Hood \& Martin, 2005).

Finalmente, la Gradación es el subsistema que permite analizar el modo en que los escritores/hablantes gradúan los significados interpersonales e ideacionales a través de dos escalas de intensidad: fuerza (referida a la manera en que el hablante aumenta o disminuye la intensidad de la valoración con un amplio rango de categorías semánticas) y foco (relacionado con el modo en que se agudiza o suaviza una valoración). Lo interesante del enfoque es que incluye valores que pueden expresarse de manera explícita por medio de un ítem léxico (como 'muy' o 'de alguna manera') pero también se considera la escala implícita, es decir, cómo el hablante selecciona el ítem léxico de acuerdo a un continuum que puede ir del grado más alto al más bajo (como 'excelente' -'muy bueno' - 'bueno'). De acuerdo con esto, se observa cómo la gradación opera trans-sistemáticamente, y en rigor, alguna escala de intensidad (de bajo a alto) es parte integrante del significado. Hood y Martin (2005) destacan la importancia de este subsistema, puesto que permite mostrar el modo en que los autores construyen una postura 'que naturaliza la crítica objetiva' a través de estos recursos.

Para el análisis de los intercambios que se producen en las devoluciones escritas y para determinar las relaciones de poder o solidaridad que allí se entretejen, nos interesa particularmente atender a la polaridad y la modalidad de las aserciones y las propuestas de los docentes, es decir que importa analizar el sistema de Compromiso para observar el grado de apertura a la negociación que el docente imprime a sus enunciados, si se presenta como un sujeto abierto a la revisión de sus posiciones (heteroglosia) o si toma distancia, cerrando el diálogo (monoglosia). Complementariamente, se consideran los recursos que se encuadran en el sistema de Gradación ${ }^{1}$. 


\section{Marco metodológico}

El estudio, que se inscribe en una investigación mayor, responde a un abordaje de tipo cualitativo, de corte exploratorio. La población participante está compuesta por 25 docentes universitarios que dictan asignaturas avanzadas de 5 carreras de grado de una universidad pública argentina, a razón de 5 profesores por carrera. En esas materias, los docentes solicitan trabajos escritos que responden a géneros disciplinares similares a los frecuentados por los graduados, como proyectos de investigación, artículos, ponencias o, según la carrera, proyectos de corte profesional, como los industriales o de intervención pedagógica o social. Estos trabajos son luego evaluados, comentados y devueltos a los estudiantes para la realización de ajustes antes de su entrega final. En ese marco, se recolectaron 184 devoluciones escritas (DE) que los profesores conservaban archivadas. Para este artículo se construyó un sub-corpus de $100 \mathrm{DE}$, seleccionando al azar 4 DE por cada participante, de modo de obtener una muestra balanceada. Las DE fueron segmentadas en CE y se obtuvieron 1010 comentarios. Para su delimitación se tuvo en cuenta un criterio gráfico, su disposición en la superficie de la hoja o la pantalla. Si bien entendemos que todos ellos constituyen expresiones multimodales porque en su construcción intervienen distintos componentes que portan significados (colores, tipografías, ubicación en la página), para este trabajo solo consideramos aquellos en los que predominaba el sistema semiótico (Matthiessen, 2009) verbal. Estos sumaron 886 CE, lo que representa un $87,72 \%$ de la muestra.

Los CE fueron transcriptos en planillas y, a partir de un examen exploratorio inicial, de tipo inductivo, se buscó identificar distintas ED empleadas por los docentes. Para la definición de las ED procuramos establecer criterios precisos con el objetivo de distinguir una de otra, tarea que no resultó sencilla por la superposición de significados que se realizan en los CE y por la complejidad de las relaciones interpersonales que en ellos se juega. En este sentido, buscamos atender a diversos componentes léxico-gramaticales (Halliday, 1994) y discursivos (Martin \& Rose, 2003; Martin \& White, 2005) que se conjugan en su organización interna para el logro simultáneo de los propósitos pragmáticos. Para el reconocimiento y análisis de las ED (Menéndez, 2005) empleadas por los docentes consideramos: a) la identificación del objeto de evaluación, b) el tipo de evaluación (positiva o negativa), c) las relaciones intersubjetivas experto-novato que se construyen y d) los recursos léxico-gramaticales y discursivos que intervienen en su construcción.

Este análisis detallado de los CE dio lugar a un esquema de análisis consistente en ocho ED: 'Aceptar', 'Rechazar', 'Ajustar', 'Problematizar', 'Empujar', 'Sugerir lecturas', 'Solicitar ampliación de la información' y 'Guiar la producción escrita' (Natale, 2013). El orden de enumeración de las ED encontradas responde al siguiente criterio: las dos primeras son evaluaciones predominantemente monoglósicas (Martin \& White, 2005), es decir que dejan escaso o nulo margen a la negociación. 'Aceptar' conlleva una 
evaluación positiva y 'Rechazar', una negativa, como se verá más adelante. Las dos que siguen acarrean evaluaciones negativas, pero admiten algún tipo de apertura. Las cuatro restantes tienen como finalidad principal ofrecer retroalimentación acerca de la organización genérica y discursiva del escrito entregado antes que expresar una evaluación de los aprendizajes conceptuales.

En una etapa posterior, nos concentramos en el examen de aquellas ED que tienen como propósito central expresar una evaluación negativa acerca del conocimiento de las teorías y conceptos estudiados, sobre la metodología empleada por los estudiantes y sobre la construcción de argumentos y conclusiones. En breve, nos focalizamos en el análisis de los recursos seleccionados por los docentes para la construcción de las ED 'Rechazar', 'Ajustar' y 'Problematizar'. Para este análisis, se han tomado del corpus ejemplos ${ }^{2}$ representativos de cada una de las estrategias.

\section{Resultados}

\subsection{Rechazar}

La estrategia que hemos denominado Rechazar consiste, en términos de Halliday (1994), en una respuesta 'no esperada' de parte del docente, ya que porta una evaluación negativa. La entidad evaluada puede ser un concepto teórico o bien un dato fáctico, es decir unidades menores del conocimiento disciplinar. La evaluación, que se inscribe en la escala de lo correcto/incorrecto, puede aparecer inscripta (expresada abiertamente) o ser evocada (sugerida) mediante distintos recursos. En este último caso, la evaluación deberá ser inferida (Sperber \& Wilson, 1986) por el lector.

Desde la perspectiva de la Teoría de la Valoración (Martin \& White, 2005), la estrategia 'Rechazar' realiza mediante recursos del sistema de Compromiso, como un recurso que produce una contracción dialógica, más específicamente como Rechazo ${ }^{3}$ (Disclaim), categoría en la que se incluyen significados en los que las alternativas dialógicas son rechazadas o son representadas como inviables (Martin \& White, 2005). 'X es incorrecto' o 'X está mal', es decir, cláusulas declarativas construidas con procesos relacionales intensivos atributivos (Halliday, 1994) en las que los atributos portan la valoración negativa podrían ser consideradas como las formas abiertas típicas del rechazo. Sin embargo, en general, las evaluaciones negativas del corpus no se manifiestan tan explícitamente.

A continuación, consideraremos distintas realizaciones de la estrategia 'Rechazar' para sistematizar los recursos semióticos, léxico-gramaticales y discursivos empleados en su construcción. Esto también permitirá reconocer que las relaciones entre los interlocutores pueden configurarse de distinta manera. 
La estrategia 'Rechazar' puede expresarse con una marca gráfica, generalmente una cruz $(\mathrm{X})^{4}$, en una sola cláusula (1) o en una estructura que comprende más de una y que da lugar a distintos movimientos, como veremos más adelante.

(1) [el fenómeno de rectificación] "fenómeno" no es la palabra. Ing-A2-4

En (1), el rechazo tiene como objeto el léxico especializado. Los estímulos ostensivos (Sperber \& Wilson, 1986) realizados por los docentes estarían entonces dirigidos a indicar la revisión de las definiciones de los términos empleados en sus proposiciones.

Otras realizaciones más complejas de la estrategia comprenden más de una cláusula. Las que acompañan a la primera, que es la que suele realizar el rechazo, cumplen otras funciones de habla, útiles para reforzar el entramado de las relaciones interpersonales. El ejemplo (2) puede servir para ilustrar lo antedicho:

(2) [Las islas denominadas como Bajo Delta, ubicadas a $20 \mathrm{~km}$ al norte de la Ciudad de Buenos Aires corresponden a la porción final de la región del Delta del Río Paraná]. Las islas no están a $20 \mathrm{~km}$ de la ciudad de Buenos Aires. Si se refieren a la $C A B A$, entonces, aclárenlo, pero una correcta interpretación de ciudad abarcaría capital y el resto de los partidos ya que es un continuo urbano. Ec-A1-11

En (2), el subrayado realizado por el docente delimita con claridad el segmento rechazado: una cláusula hipotáctica no finita con información geográfica. En cuanto a los significados expresados en el comentario encontramos que se distribuye en distintas cláusulas y que se organiza en distintas fases (Gregory, 2002). Al igual que otros ejemplos del corpus, encontramos una estructura que en su realización completa se compone de tres fases (ver Tabla 1): la Evaluación, los Fundamentos (de la evaluación) y la Orientación (de la acción), por lo que la hemos denominado EFO.

Tabla 1. Ejemplos de las fases de la estructura EFO en una estrategia.

\begin{tabular}{|l|l|}
\hline \multicolumn{1}{|c|}{ Cláusula } & \multicolumn{1}{c|}{ Fases } \\
\hline Las islas no están a 20 km de la ciudad de Buenos Aires. & Rechazar/Evaluación \\
\hline Si se refieren a la CABA, entonces aclárenlo, & Orientación \\
\hline $\begin{array}{l}\text { pero una correcta interpretación de ciudad abarcaría capital y el } \\
\text { resto de los partidos }\end{array}$ & Fundamento 1 \\
\hline ya que es un continuo urbano. & Fundamento 2 \\
\hline
\end{tabular}

La primera cláusula, una aseverativa polar negativa, realiza el rechazo propiamente dicho. Para ello, se retoma una afirmación de los estudiantes y se invierte su polaridad, lo que evoca una evaluación negativa ('afirmación incorrecta') por la presencia del adjunto modal 'no', que se trata de un caso de uso del 'no' evaluativo de tipo correctivo que cumple "la función pragmática de indicar lo que no está correcto y que necesita ser solucionado o mejorado" (Bolívar, 2011: 70). Si bien se reconoce que la negación implica asumir un posicionamiento dialógico (Martin \& White, 2005), la 
proposición no es modalizada. Por ello, si bien incluye en sí misma la posición contraria, no parece haber en esa unidad gramatical un margen de negociación sobre los significados que se intercambian. No obstante, las cláusulas subsiguientes abren paso a la consideración de otras posiciones. En primer lugar, la cláusula que conlleva el rechazo propiamente dicho es seguida por un complejo clausal (b) que se inicia con una cláusula condicional hipotáctica. En ella, el docente reconoce que los estudiantes han tenido en cuenta una información, aunque no la han explicitado. Asimismo, la construcción de la cláusula como una condicional indica, por un lado, que lo que el profesor expresa es una posibilidad entre otras. Por esto, la condicional, tal como está planteada, abre el juego de la negociación (Martin \& Rose, 2003). La cláusula principal que sigue es una orden congruente, con imperativo, sobre una norma académica referida la precisión en el uso del lenguaje. Entendemos que estas órdenes (sean polares o moduladas) constituyen un movimiento interno a la estrategia, una Orientación para los estudiantes, es decir, una guía para salvar el error.

Las dos cláusulas que siguen ( $\mathrm{d}$ y e) contienen los Fundamentos; se trata de aseverativas que funcionan como argumentos de apoyo a la evaluación negativa y/o para justificar la Orientación. En (2), específicamente, el docente ofrece información que parece no haber sido comprendida por los estudiantes: el concepto de ciudad o el entramado urbano en el que se inserta la ciudad. De este modo, las cláusulas que siguen a la primera (la polar negativa), permiten entender que lo que el docente está rechazando no es una información referida a un dato fáctico, la distancia entre dos puntos geográficos, sino el modo de conceptualizar la ciudad.

Una cuestión no menor que surge de (2) es el modo en que se articulan las distintas cláusulas que componen el comentario. Se establecen entre ellas distintos tipos de relaciones lógico-semánticas, explícitamente indicadas por medio de los conectores 'si', 'pero' y 'ya que', que se corresponden con relaciones condicionales, adversativas y causales, todas en muy pocas líneas. Esta compleja articulación del comentario, a pesar de su brevedad, da cuenta del dialogismo implicado en la interacción. El reconocimiento de una posición diferente de la de la voz autoral se señala mediante la construcción adversativa que se inicia con 'pero' y por la justificación de las propias aseveraciones. Además, cabe notar que la cláusula c), que brinda información sobre cómo conceptualizar la ciudad, es modalizada, se construye con un verbo en potencial ('abarcaría'), lo que también revela la apertura a la heteroglosia (Martin \& White, 2005). Por otro lado, las relaciones lógico semánticas y la utilización de los conectores evidencian el carácter discursivo de los CE.

La puesta en juego de los recursos mencionados da lugar a la construcción de relaciones interpersonales que conjugan el poder y la solidaridad. Esto se da en dos movimientos: la primera cláusula no habilita la negociación de la información, cierra el espacio con un rechazo explícito. En la segunda, la posición de poder del docente se 
mantiene, especialmente por el uso del imperativo, al que Martin y White (2005) encuadran como un recurso de la monoglosia. Sin embargo, más allá de la distancia, el profesor brinda una orientación y, además, en las cláusulas subsiguientes ofrece información que, aparentemente, juzga como no comprendida para apoyar tanto su rechazo como la orden. Más allá de esto, el solo esfuerzo de brindar los datos que fundamentan el rechazo trasluce su intención de ser solidario con los estudiantes. Aunque muy poco frecuentes, otras expresiones verbales de la estrategia 'Rechazar' expresan más abiertamente la evaluación negativa y adoptan una posición más distante, más asimétrica con los destinatarios, como se ve en (3):

(3) [El municipio no cuenta con la implementación del Plan Argentina Trabaja]

a) error!

b) Recuerden que hasta les sugeri que entrevistaran al funcionario del MDS que está a cargo! Ec-A1-5

Como podemos observar aquí la estrategia 'Rechazar' tiene también una estructura compuesta, ya que se articula en dos cláusulas: 1) una menor que porta la evaluación negativa y 2) una segunda que adopta la construcción gramatical de una orden de grado alto por el uso del imperativo, pero que indirectamente expresa un Fundamento de la evaluación negativa, una prueba de que los estudiantes no siguieron una sugerencia. Tal vez la selección de un recurso valorativo tan taxativo se deba a la inclusión de una información falsa y al incumplimiento de una sugerencia previa del docente.

En síntesis, a partir del relevamiento y el examen de distintos ejemplos de la estrategia 'Rechazar' encontramos que, dentro del conjunto de las manifestaciones verbales, existen distintos grados de desarrollo de la estrategia. El nivel más básico está dado por una cláusula menor como 'Error!' o 'No!'. También hallamos cláusulas polares negativas, construidas con procesos relacionales atributivos intensivos en las que el Portador o Carrier puede ser un concepto o término disciplinar o bien una cláusula completa. La evaluación o el rechazo residen, precisamente, en la polaridad negativa que actúa como contracara de la aserción de los estudiantes. Por otro lado, algunas realizaciones de la estrategia muestran una organización más compleja, con articulación de varias cláusulas. En ellas, si bien se rechaza el uso de un concepto, un término o un dato fáctico, luego se brindan argumentos para sostener el rechazo, así como orientaciones para resolver el error.

Así, de acuerdo con nuestro análisis, las realizaciones de la estrategia 'Rechazar' entraman de distintas maneras las relaciones de poder y de solidaridad entre expertos y novatos, las que pueden situarse en un continum. El uso de recursos gráficos, que no analizamos aquí por razones de espacio, aparece como la expresión que establece mayor distancia entre el docente y los estudiantes. El profesor no dedica gran esfuerzo a explicar cuál es el error encontrado, sino que se limita a dejar asentado que encontró 
alguno. La distancia intersubjetiva se construye también en los casos en que los docentes eligen hacer explícita la evaluación negativa. Mayor solidaridad encontramos en las realizaciones que combinan marcas visuales y palabras y en las exclusivamente verbales. Esta relación se construye, en principio, a partir del hecho del tiempo dedicado a la formulación del rechazo. Además, cuando los CE incluyen otros segmentos que sirven para mitigar las evaluaciones negativas y/o para orientar el futuro desempeño de los estudiantes, mayor es el grado de cooperación que se establece. No obstante, todas las variantes de la estrategia 'Rechazar' implican una clara demarcación de que algunos significados no son negociables, como el léxico, los conceptos disciplinares y la información fáctica. En este sentido, el conocimiento disciplinar aparece como un bien que se posee o no se posee y, si no se posee, impone la puesta de un límite al ingreso a la comunidad disciplinar por parte del docenteexperto. Así, esta figura emerge como un gatekeeper (Berkenkotter \& Huckin, 1995), alguien que tiene la potestad de abrir o cerrar las puertas al novato. Este, a su vez, aparece como alguien que aún no ha alcanzado el nivel de conocimiento suficiente como para ser admitido.

\subsection{Ajustar}

Esta estrategia, al igual que la anterior, acarrea una evaluación negativa acerca de un aspecto relativo a la investigación científica, actividad a la que apuntan algunos de los géneros que se demandan en las últimas materias de las carreras de grado, como proyectos de investigación y tesinas. Las escalas de evaluación, generalmente implícitas, son las de la especificidad y la precisión, características que se asocian a las actividades y al lenguaje de las ciencias. Si bien la estrategia implica una respuesta no esperada (Halliday, 1994), no llega a constituir un rechazo, ya que la dada por el novato no es plenamente negada o calificada como un error, como ocurre en los ejemplos analizados en el apartado anterior. Antes que eso, el docente indica que es necesario delimitar o acotar el alcance de una expresión, como puede ser un interrogante de investigación, la formulación del problema o la presentación del objeto de estudio.

Los recursos léxico-gramaticales que pueden ser empleados en la realización lingüística de la estrategia son variados, como se verá en el análisis de (4), (5) y (6):

(4) [se analizará cómo el discurso mediático puede influir en las manifestaciones urbanas populares] En su organización? En su cobertura? En su recepción? EP-A3-1.

En este comentario, compuesto por tres preguntas, la docente busca mostrar que el problema que el estudiante pretende abordar en su trabajo no ha sido claramente delimitado y que no logra el nivel de especificidad requerido en una investigación. Los interrogantes que realizan la estrategia dan cuenta de distintos ejes que podrían tratarse. Por otro lado, podemos pensar que la observación porta significados que se 
corresponden con dos planos: el trabajo del estudiante y el método científico, según el cual un problema debe estar definido con precisión y examinado en unidades simples. El método científico es también objeto de evaluación en (5):

(5) [¿Está funcionando adecuadamente el primer nivel de atención en el distrito de San Miguel, en la actual gestión municipal?] Las preguntas no responden exactamente a la manera en la que está formulado el problema. PS-A1-11

Como podemos observar, la docente no rechaza de plano los interrogantes. Estos no aparecen como incorrectos o incoherentes o incompletos, sino como 'algo desajustados'. Así, lo que requiere una revisión es la formulación de las preguntas. El recurso que, unido a la polaridad negativa, permite reconocer la estrategia es 'exactamente' que se encuadra entre los de la gradualidad, y más específicamente en los relativos al Foco (Hood \& Martin, 2005; Martin \& White, 2005). Lo que se pretende señalar, precisamente, es que la formulación de los estudiantes es presentada a través de un foco 'borroso' o difuso (Hood \& Martin, 2005). En (6), la estrategia recae sobre otro aspecto de la investigación: el objeto de análisis:

(6) [Establecer la secundaria como obligatoria: 6 años primaria y 6 secundaria.] Esto en provincia. Ed-P1-14

En este caso, la docente realiza una afirmación que deja entrever que el estudiante no ha sido exhaustivo en el tratamiento del objeto de estudio. La observación es formulada de manera indirecta. La profesora se limita a señalar que la información proporcionada es válida solo para el caso de una jurisdicción educativa. Para poder ajustar el texto, el estudiante deberá inferir dos cuestiones: en qué radica su error y qué otros datos deben incluir para subsanarlo.

Los ejemplos examinados ubican al docente en el rol de un lector conocedor de la disciplina, de sus objetos de estudio y de sus metodologías de investigación. Como formador, demanda especificaciones a un destinatario que aparece posicionado en un rol de inferioridad. La situación podría asimilarse a la que se genera en instancias de evaluación de proyectos de investigación. En este sentido, en estos casos el estudiante tiene la posibilidad de participar en procesos propios de la vida académica durante su formación de grado. Más allá de las similitudes en el contexto que se crea en ambos $\mathrm{CE}$, existen algunas diferencias en cuanto al grado de cooperación que el experto muestra en cada caso. En el ejemplo (4) organizado con tres preguntas, el docente no solo sugiere que la formulación del problema no es lo suficientemente precisa, sino que abre un abanico de opciones de posibles abordajes de la problemática planteada. En este movimiento, las distancias entre él y el novato se ven reducidas. En cambio, en (5), el experto reclama el ajuste en un aspecto, la formulación de las preguntas, pero no da orientaciones concretas. Por último, en el tercer caso, la docente opta por no señalar que hay una falta de precisión, solo marca que la afirmación del estudiante solo 
refleja una parte del objeto de análisis. El estudiante deberá entonces inferir que la información que maneja no agota la requerida.

En este sentido, el docente deja entrever que la investigación y la producción escrita académica constituyen tareas que requieren de una sucesión de ajustes, una especie de obra en progreso que puede ser mejorada a partir de la interacción con un docente experto que se constituye en guía del estudiante. A su vez, tanto el profesor como el alumno son mostrados como seres activos y mutuamente involucrados en una labor conjunta, la producción y la comunicación de conocimientos generados en la investigación.

\subsection{Problematizar}

La estrategia que llamamos 'Problematizar' tiene como objeto una aserción o un conjunto de aserciones propias del proceso de investigación, como la formulación de una hipótesis, de una interpretación o una conclusión. También se puede referir a una de las instancias metodológicas, como la propuesta de una herramienta de análisis. Implícitamente, desde su rol de experto en el campo, el docente realiza una evaluación negativa de la escala de lo cuestionable/incuestionable o discutible/ indiscutible ya que, precisamente, la estrategia consiste en poner en cuestión las afirmaciones del novato. Sin embargo, el experto opta por no rechazarlas abiertamente, de allí que no explicite sus apreciaciones ni utilice construcciones polares negativas. En cambio, selecciona recursos que le permiten mitigar su devolución $\mathrm{y}$, a la vez, ofrecer alternativas que le sirvan para repensar sus interpretaciones.

Algunas realizaciones servirán para ilustrar lo antedicho. En (7) y (8) la aserción problematizada es recuperada en términos casi textuales, pero el docente la reformula introduciendo un cambio en el plano de la modalidad. Emplea para ello adjuntos modales que afectan a la totalidad del enunciado:

(7) Respecto a los modelos de pedagógicos (de la transmisión, del adiestramiento y de la problematización) utilizados para la capacitación del personal de la salud, se puede identificar en el Programa REMEDIAR al modelo de la problematización]. Están seguros que estamos frente a un modelo de problematización en las capacitaciones del Remediar? Si es así, justifiquen por qué elementos de estas capacitaciones se ve que es un modelo del tipo de problematización. PS-A1-19

(8) Por el otro lado nos encontramos con el tema de la reproducción masiva de madres de más de siete hijos situación que nos lleva a ver cómo el accionar de las personas cambia según los distintos contextos sociales] mi duda es si cambia el accionar o buscan las posibilidades que da el Estado en el marco de sus situaciones. PS-P221 
La modalización del enunciado de los estudiantes se plantea en relación con su grado de certeza, es decir en la modalidad epistémica y se refuerza, además, por los recursos léxicos seleccionados para construir los adjuntos modales. En ambos casos, la falta de certeza se lexicaliza en una expresión verbal ('estar seguro') incluida en una interrogativa o en un sustantivo ('duda').

Ambos ejemplos, por otro lado, recurren a otro recurso de la problematización. Este consiste en lo que Hyland y Hyland (2006) llamaron la estrategia de atribución personal (personal attribution) según la cual los docentes adoptan una voz menos amenazante para su imagen y se muestran como lectores ordinarios antes que como expertos.

La atribución personal se manifiesta de distinta manera en (7) y (8). En el primero, el experto se ubica como par de los novatos al utilizar la primera persona del plural ('Están seguros de que 'estamos' en presencia....?'). Así, construye para sí la imagen de una persona que, en tanto par, confiará en lo que sus compañeros establezcan o que, en todo caso, corre los mismos riesgos si hay un error. En el segundo, la atribución personal aparece más claramente marcada en el pronombre posesivo de primera persona ('mi' duda). Por otro lado, esta primera persona discursiva se representa a sí misma como alguien que no posee plenamente un saber, que tiene dudas. De esta manera, evita emitir una apreciación directa sobre los planteos de la estudiante y pone a resguardo tanto su propia imagen como la de los novatos.

Por otro lado, encontramos en los mismos ejemplos la apelación al recurso de la interrogación, directa en la primera cláusula de (7) e indirecta en (8). Estas preguntas pueden ser interpretadas de dos maneras: como manifestación de la (supuesta) falta de certeza acerca de lo que se discute o como preguntas retóricas. Esta última posibilidad se articula con otro recurso, la conexión de oposición que se da entre dos opciones. De esta manera, lo que es presentado como una duda puede ser leído como la puesta en consideración de otra lectura, que aparece como la que el docente defiende. En (8) podemos notar dos interpretaciones sobre un hecho, la de la estudiante y la de la docente. El docente plantea su duda acerca de cuál de las dos lecturas es la mejor.

Sin embargo, más allá de las distintas claves lingüísticas que se encuentran en (8), la posición sostenida por el docente no es explicitada, debe ser inferida por sus destinatarios, es decir que en esta estrategia el profesor no toma la opción de manifestar abiertamente su opinión. Este hecho se relaciona, precisamente, con lo que aparece como el eje central de la estrategia: impulsar la exploración de otras posibilidades de interpretación de un dato relevado o de la lectura crítica de una fuente. A diferencia de la estrategia 'Rechazar', en la problematización, el experto no clausura totalmente la aserción del estudiante, sino que la somete a discusión confrontándola con su propio análisis. 
Las realizaciones de la estrategia analizadas hasta aquí muestran entonces que una primera fase consiste en poner en cuestión una aseveración de los estudiantes, mediante su modalización en lo que se refiere a la certeza, lo que se lleva adelante mediante distintos recursos léxico-gramaticales que suelen amalgamarse:

- la introducción de un adjunto modal que afecta a una aseveración de los estudiantes en su conjunto, la que es retomada por el docente,

- la transformación de la aseveración de los estudiantes en una pregunta, lo que implica la transformación de la función de habla original,

- el establecimiento de una relación de oposición entre la aseveración de los novatos y otra interpretación.

Pero este primer paso se complementa muchas veces con otro que expresa una orientación. Esta puede asumir distintos grados de obligación, desde los más altos, expresados abiertamente, con un imperativo, hasta formas muy indirectas, expresadas como preguntas o pedidos. Estas orientaciones, cuando están, solicitan la fundamentación de las aserciones, un desarrollo de la argumentación. En (7), esto está claramente expresado en el verbo 'justificar' en imperativo. Estas órdenes o pedidos pueden ser también interpretados como complementos para apoyar la modalización sobre su grado de certeza; la orden está sujeta a la posibilidad introducida por la cláusula condicional que la precede.

Luego de haber observado las realizaciones léxico-gramaticales y discursivas de la estrategia 'Problematizar', resulta pertinente que consideremos las relaciones que se establecen entre los participantes del intercambio. En primer lugar, debemos señalar que esta estrategia se inscribe claramente entre las categorías heteroglósicas del sistema de compromiso de la Teoría de la Valoración. La utilización de los recursos de la modalidad epistémica permite ubicarla como una estrategia de apertura dialógica. Guarda algunas similitudes con la categoría denominada Consideración (Entertain) por Martin y White (2005). Según los autores, la apertura dialógica señalizada por la modalización crea un espacio para voces alternativas, que pueden estar incluso en conflicto con la sostenida por la voz autoral. A través del reconocimiento de otras posiciones, a la vez, se establece una relación de solidaridad con los destinatarios, ya que: "aquellos que sostienen posiciones contrarias son reconocidos como potenciales participantes del coloquio en curso" (Martin \& White, 2005: 109). Esta posibilidad resulta particularmente relevante en el contexto de las interacciones que estamos analizando, ya que la apertura del espacio de diálogo y de discusión sobre los hallazgos de los estudiantes puede resultar estimulante para los novatos, en tanto implica un reconocimiento. Sin embargo, aunque los docentes asuman una posición de solidaridad, no declinan el poder que les confiere su rol. Por el contrario, este aparece también representado en el lenguaje, más evidentemente por las órdenes que suelen 
seguir a la problematización propiamente dicha pero también en el solo hecho de plantear dudas sobre las aseveraciones de los estudiantes.

En estos ejemplos, la producción académica es mostrada en su dimensión procesual, como una actividad que apunta a la creación de conocimiento a partir de la interacción entre actores sociales interesados en el fenómeno que se está analizando. Esta construcción surge del hecho que una parte del texto o una afirmación es tomada como objeto de discusión. Tras la devolución del experto, el novato puede optar por introducir en su escrito modificaciones que, implícitamente, le están siendo sugeridas. Así, el texto es mostrado como una entidad que se encuentra en desarrollo.

El conocimiento disciplinar se presenta como un campo abierto a la discusión, que se construye colectivamente a partir de la interacción entre los miembros de una comunidad y no como un saber ya construido. Por otro lado, en esta estrategia se manifiestan algunos rasgos del proceso de creación de conocimiento, como la problematización de una interpretación, la interrogación y la exploración de distintas hipótesis.

En cuanto a los participantes del intercambio, puede decirse que el docente aparece como un experto que se ubica en el rol de un sujeto que, antes que rechazar de plano una idea o afirmación, decide someterla a discusión. Este hecho, a su vez, ubica al estudiante en un rol activo, como el de quien debe continuar avanzando en su proceso de formación y de elaboración del conocimiento, para lo que se le ofrece una guía. En este sentido, se construyen entre ellos relaciones que dan cuenta, a la vez, del poder del docente y de su solidaridad para con el aprendiz.

\section{CONCLUSIONES}

En este artículo nos hemos concentrado en la caracterización de tres ED empleadas en CE de docentes universitarios para expresar evaluaciones negativas sobre los conocimientos disciplinares, las metodologías empleadas y sobre los argumentos y conclusiones que elaboran estudiantes de distintas carreras de grado de una universidad pública argentina. Estas estrategias, 'Rechazar', 'Ajustar' y 'Problematizar', fueron identificadas en un estudio exploratorio basado en un corpus de 1010 CE incluidos en DE producidas en el marco de asignaturas avanzadas de distintas carreras de grado. Para la distinción entre las ED se contempló el objeto de evaluación, la escala aplicada, el tipo de relaciones intersubjetivas experto-novato que se construyen, considerando los recursos del sistema de Compromiso, y la organización de los CE en fases.

El análisis cualitativo nos permitió establecer diferencias entre las tres ED que construyen evaluaciones negativas. Encontramos que la estrategia 'Rechazar' se emplea para evaluar conocimientos fácticos o nociones básicas de la disciplina, a partir de la escala correcto/incorrecto. En sus realizaciones verbales, se hallan 
prioritariamente cláusulas polares negativas, con un 'no' evaluativo correctivo (Bolívar, 2011). En el sistema de Compromiso de la Teoría de la Valoración, este tipo de construcción se corresponde con la categoría Rechazo (Disclaim), que señala un límite para admitir la viabilidad de una afirmación. En este sentido, se produce una contracción dialógica y, por tanto, una distancia interpersonal entre el docente y el estudiante. El cierre a la negociación se produce frente a la constatación del docente de la falta de aprendizaje de aspectos (datos o conceptos) que aparecen como de bajo nivel de dificultad o que indicarían la falta de estudio de los estudiantes. Así, la distancia entre el experto y el novato se realiza mediante construcciones lingüísticas específicas, como las cláusulas polares negativas. No obstante, en algunas realizaciones de la estrategia se encuentra que los docentes, una vez establecido el error, optan por mostrarse cooperativos fundamentando sus evaluaciones negativas y ofreciendo lineamientos para subsanarlo, como se observó en la descripción de la estructura EFO.

En cuanto a la estrategia 'Ajustar', se trata también de una evaluación negativa, en la escala de lo preciso/impreciso. Es empleada en comentarios referidos a aspectos vinculados con los géneros vinculados con la iniciación en la investigación, como la delimitación del problema y del objeto de estudio o la formulación de los interrogantes. Las evaluaciones no son expresadas de manera abierta, como en 'Rechazar', sino que prevalecen otras opciones discursivas. Si bien en algunos casos se verifica la presencia de un 'no' correctivo, este está matizado a través de recursos de la Gradualidad, como en la expresión 'no exactamente', que señaliza la falta de precisión en las producciones estudiantiles. En otros casos, los docentes demandan mayor precisión a partir de un conjunto de interrogaciones que ofrecen al estudiante varias opciones. En movimientos de este tipo, el docente se construye como un sujeto solidario que, antes que marcar errores de manera explícita, deja entrever que se espera que el estudiante atienda a la precisión del lenguaje disciplinar.

La estrategia 'Problematizar', por su parte, revela un mayor grado de acercamiento entre el experto y el novato. Como hemos observado, se aplica a cláusulas que dan cuenta de la progresiva apropiación de los procesos de construcción del conocimiento disciplinar por parte de los estudiantes. En efecto, es una estrategia destinada a expresar evaluaciones negativas sobre la formulación de resultados y conclusiones de las investigaciones llevadas adelante por los novatos. En estos casos, los docentes construyen sus evaluaciones apelando a recursos del sistema de Compromiso que se clasifican como Consideración (Entertain), con un grado de apertura importante a posiciones diferentes de las sostenidas por un hablante. En este sentido, el conocimiento disciplinar aparece como una entidad inacabada, abierta a los aportes de los futuros miembros de la comunidad. No obstante, también se encuentra que los docentes no ceden su poder, ya que hallamos que demandan de parte de los estudiantes la justificación de las aserciones vertidas. 
En general, la caracterización de las ED que expresan evaluaciones negativas sobre los avances en el conocimiento de los conceptos y de las epistemologías disciplinares da cuenta de distintos tipos de relaciones intersubjetivas entre expertos y novatos. Hemos observado que los docentes se muestran más cerrados a la negociación y, por ende, más distantes, cuando los estudiantes no logran dar cuenta del aprendizaje de aspectos básicos de los campos del saber a los que aspiran a ingresar. Sin embargo, en la medida en que ensayan el aprendizaje de cuestiones relativas a la investigación y a la producción de conocimiento disciplinar, los profesores se abren a la negociación de posiciones y se muestran más solidarios, dando lugar a los novatos a continuar discutiendo los avances realizados.

Entendemos que estos hallazgos ofrecen un panorama que puede resultar útil a docentes que buscan mejores maneras de retroalimentar los procesos de producción científica en sus estudiantes y a los especialistas en alfabetización académica (Carlino, 2013). Asimismo, pueden constituir un aporte para el estudio de la evaluación en el medio académico, a partir de un género que no ha merecido hasta el momento suficiente atención, al menos desde el análisis del discurso. El estudio constituye una exploración que requiere, en principio, de una ampliación del corpus. Las devoluciones examinadas fueron producidas en un contexto institucional particular y, por ello, las ED encontradas pueden obedecer a aspectos culturales o ideológicos de la propia universidad no considerados en este análisis. Por ello, quedan abiertas las puertas a nuevos estudios que profundicen el análisis sobre la evaluación negativa en el contexto académico en general y en la educación superior en especial, de modo de producir conocimientos que cooperen con la formación de las futuras generaciones de expertos disciplinares.

\section{REFERENCIAS BIBLIOGRÁFICAS}

Arancibia Gutiérrez, B., Tapia-Ladino M. \& Correa Pérez, R. (2019). La retroalimentación durante el proceso de escritura de la tesis en carreras de pedagogía: Descripción de los comentarios escritos de los profesores guías. Revista Signos. Estudios de Lingüistica, 52(100), 242-264.

Berkenkotter, C. \& Huckin, T. (1995). Genre knowledge in disciplinary communities. Hillsdale, HJ: Lawrence Erlbaum.

Bolívar, A. (2008). El informe de arbitraje como género discursivo en la dinámica de la investigación. Revista Latinoamericana de Estudios del Discurso, 8(1), 41-64.

Bolívar, A. (2011). Funciones discursivas de la evaluación negativa en informes de arbitraje de artículos de investigación. Núcleo, 28, 59-89.

Brown, P. \& Levinson, S. C. (1987). Politeness: Some universals in language usage. Cambridge: Cambridge University Press. 
Carlino, P. (2013). Alfabetización académica diez años después. Revista mexicana de investigación educativa, 18(57), 355-381.

Gregory, M. (2002). Phasal analysis within communication linguistics: Two contrastive discourses. En P. H. Fries, M. Cummings, D. Lockwood \& W. Spruiell (Eds.), Relations and Functions within and around Language (pp. 316-545). Nueva York: Continuum.

Gumperz, J. J. (1982). Discourse strategies. Cambridge: Cambridge University Press.

Halliday, M. A. K. (1978). El lenguaje como semiótica social. México: FCE.

Halliday, M. A. K. (1994). An Introduction to functional grammar. Londres: Edward Arnold.

Hood, S. \& Martin, J. R. (2005). Invocación de actitudes: El juego de la gradación de la valoración en el discurso. Revista Signos. Estudios de Lingüística, 38(58), $195-$ 220.

Hunston, S. \& Thompson, G. (2000). Evaluation in text: Authorial stance and the construction of discourse: Authorial stance and the construction of discourse. Oxford: Oxford University Press.

Hyland, K. \& Hyland, F. (2006). Feedback in second language writing: Contexts and issues. Cambridge: Cambridge University Press.

Kaplan, N. (2004). Nuevos desarrollos en el estudio de la evaluación en el lenguaje: La teoría de la valoración. Boletín de lingüística, 22, 52-78.

Kumar, V. \& Strake, E. (2011). Examiners' reports on theses: Feedback or assessment? Journal of English for Academic Purposes, 10(4), 211-222.

Lea, M. R. \& Street, B. V. (1998). Student writing in higher education: An academic literacies approach. Studies in higher education, 23(2), 157-172.

Martin, J. R. (1992). English text. System and structure. Filadelfia/Ámsterdam: John Benjamins.

Martin, J. R. \& Rose, D. (2003). Working with discourse: Meaning beyond the clause. Londres: Continuum.

Martin, J. R. \& Rose, D. (2008). Genre relations: Mapping culture. Londres: Equinox.

Martin, J. R. \& White, P. (2005). The language of evaluation. Appraisal in English. Londres: Palgrave Macmillan. 
Matthiessen, C. M. (2009). Multisemiosis and context-based register typology: Registerial variation in the complementarity of semiotic systems. En E. Ventola \& A. Moya Guijarro (Eds.), The World Told and the World Shown. Multisemiotic issues (pp. 11-38). Londres \& Nueva York: Palgrave Macmillan.

Menéndez, S. M. (2005). ¿Qué es una estrategia discursiva? En S. Santos \& J. Panesi (Comp.), Actas del Congreso Internacional: Debates Actuales. Las teorías críticas de la literatura y la linguïstica. Buenos Aires: Facultad de Filosofía y Letras, UBA.

Natale, L. (2013). Relaciones intersubjetivas entre expertos y novatos en devoluciones escritas de docentes universitarios. Legenda, 17(16), 86-114.

Natale, L. (2014). Interrelaciones entre representaciones discursivas sobre la escritura académica y devoluciones escritas de docentes universitarios. Onomázein, Número Especial IX ALSFAL, 81-98.

Padilla, C. \& López, E. (2019). Prácticas de retroalimentación en aulas universitarias de humanidades: Comentarios digitales docentes y perfiles estudiantiles de escritor. Revista Signos. Estudios de Lingüistica, 52(100) 330-356.

Poynton, C. (1985). Language and gender: Making the difference. Geelong, Vic.: Deakin University Press.

Sperber, D. \& Wilson, D. (1986) Relevance: Communication and cognition. Oxford: Basil Blackwell.

Tannen, D. (1993). Gender and conversational interaction. Oxford: Oxford University Press.

Thompson, G. \& Hunston, S. (2000). Evaluation in text: Authorial stance and the construction of discourse. Oxford, Nueva York: Oxford University Press.

\section{* AGRADECIMIENTOS}

El artículo surge de los datos recogidos en la tesis doctoral de la autora, titulada "Representaciones sobre la escritura disciplinar y su enseñanza en el discurso de docentes universitarios", dirigida por el Dr. Salvio Martín Menéndez y radicada en la Facultad de Filosofía y Letras de la Universidad de Buenos Aires. La investigación fue desarrollada con la participación de docentes de la Universidad Nacional de General Sarmiento, que aportaron devoluciones escritas realizadas sobre los trabajos que habían solicitado en las asignaturas que dictan. La autora agradece su colaboración, así como la de los revisores anónimos, por su generosa retroalimentación. 


\section{NOTAS}

${ }^{1}$ Por razones de espacio, no desarrollamos en este apartado las categorías que Martin y White (2005) subdividen cada uno de los sistemas. En el examen de las estrategias caracterizamos aquellas que resultan necesarias a los fines del análisis.

2 Detrás de cada uno de los ejemplos incluimos el código de identificación de los docentes participantes del estudio y de los comentarios que realizaron. Estos códigos provienen de las abreviaturas con las que identificamos las 5 carreras en las que dictan clases y de los cargos que ocupan los docentes (profesor o asistente). Las carreras son: Ingeniería Industrial (Ing.) y las Licenciaturas en Educación (Ed.), Políticas Sociales (PS), Estudios Políticos (EP) y Ecología (Ec). También se indica su número de identificación personal (NIP) en el conjunto de participantes de una misma carrera. Finalmente, se incluye el número de comentario realizado por ese docente. Por ejemplo: "Ing-A2-4" indica que se trata del CE número 4, realizado por el docente asistente 2 de la carrera de Ingeniería Industrial.

${ }^{3}$ La traducción es nuestra.

${ }^{4}$ En este artículo no incluimos el análisis de realizaciones gráficas del Rechazo debido a las restricciones de espacio.

${ }^{5}$ Entre corchetes transcribimos el fragmento de la producción estudiantil que es objeto de evaluación. En cursiva, el comentario del docente. 